\title{
Eletrocardiografia em onças-pardas (Puma concolor) anestesiadas com sevoflurano ou isoflurano
}

[Electrocardiography in pumas (puma concolor) anesthetized with sevoflurane or isoflurane]

\author{
A.R. Oliveira ${ }^{1}$, K.F. Silva ${ }^{1}$, M.I.P. Palumbo ${ }^{2}$, A.I. Souza ${ }^{2}$, T.D. Souza $a^{2}$, V.B. Albuquerque ${ }^{3}$, \\ M.A. Araújo $^{2}$, F.O. Frazilio ${ }^{2 *}$ \\ ${ }^{1}$ Aluna de pós-graduação - Universidade Federal de Mato Grosso do Sul - Campo Grande, MS \\ ${ }^{2}$ Universidade Federal de Mato Grosso do Sul - Campo Grande, MS \\ ${ }^{3}$ Pesquisadora Fundect CNPq - Campo Grande, MS
}

\begin{abstract}
RESUMO
O objetivo deste trabalho foi descrever os achados eletrocardiográficos de 11 onças- pardas (Puma concolor). Os animais foram sedados com $0,15 \mathrm{mg} / \mathrm{kg}$ de detomidina associado a $5 \mathrm{mg} / \mathrm{kg}$ de cetamina e mantidos anestesiados com sevoflurano (GSEVO, $n=6$ ) ou isoflurano (GISO, $n=5$ ). A frequência cardíaca foi de $95 \pm 13 \mathrm{bpm}$. As alterações observadas nos animais no GSEVO foram: atrial standtill com condução ventricular, episódios isolados de contração ventricular prematura, bloqueio atrioventricular de primeiro grau, diminuição da amplitude do complexo QRS, onda S profunda e aumento da amplitude da onda T. No grupo GISO, observou-se bloqueio de ramo direito do feixe de His, bloqueio atrioventricular de primeiro grau e aumento da amplitude da onda T. Arritmias não puderam ser associadas ao uso dos anestésicos inalatórios devido à não sensibilização do miocárdio às catecolaminas. Achados como o BAV de primeiro grau pode ter ocorrido devido ao uso de agonistas $\alpha-2$ adrenérgicos. Este estudo aumentou o conhecimento sobre as alterações eletrocardiográficas em onças-pardas anestesiadas, entretanto mais estudos são necessários para correlacionar estes achados ao uso de agentes anestésicos.
\end{abstract}

Palavras-chave: alfa-2 agonistas, anestesia geral, anestesia inalatória, cardiologia, detomidina

\begin{abstract}
The aim of this study was to describe electrocardiographic findings in pumas (Puma concolor). The animals were sedated with $0.15 \mathrm{mg} / \mathrm{kg}$ of detomidine plus $5 \mathrm{mg} / \mathrm{kg}$ of ketamine and anesthetized with sevoflurane $(G S E V O, n=6)$, or isoflurane $(G I S O, n=5)$. The heart rate was $95 \pm 13 \mathrm{bpm}$. The changes observed on GSEVO animals were: atrial standstill with ventricular conduction, isolated episodes of ventricular premature contraction, atrioventricular blockage of first degree, reduction of the amplitude of the QRS complexes, deep $S$ wave, and increase of the amplitude of the T wave. In the GISO group a right bundle branch block of the His bundle, atrioventricular blockage of first degree and of the amplitude of the $T$ wave. Arrhythmias couldn't be related to the use of inhalant anesthesia due to the lack of myocardial awareness to catecholamines. Findings such as atrioventricular block in first degree may have occurred due to the use of $\alpha-2$ adrenergic agonists. This study improved the knowledge about electrocardiographic alterations in anesthetized pumas, however further studies are required to correlate these findings to the use of anesthetic agents.
\end{abstract}

Keywords: Alpha-2 agonists, general anesthesia, inhalation anesthesia, cardiology, detomidine

Recebido em 13 de maio de 2016

Aceito em 6 de julho de 2016

*Autor para correspondência (corresponding author)

E-mail: fabricio.frazilio@ufms.br 


\section{INTRODUÇÃO}

O estudo dos traçados eletrocardiográficos em grandes felídeos é escasso na literatura, o que leva à dificuldade de identificação dos valores de referência na espécie e, consequentemente, à dificuldade diagnóstica de anormalidades cardíacas nesses animais (Oda et al., 2007; Larsson et al., 2008).

O eletrocardiograma (ECG) é um exame que pode ser utilizado em avaliações clínicas na medicina veterinária. É considerado o padrãoouro para o diagnóstico não invasivo de arritmias e distúrbios de condução por fornecer informações a respeito de dilatações e hipertrofias cardíacas (Goodwin, 2002), assim como para sugerir alguns desequilíbrios eletrolíticos, como de cálcio e potássio, e ainda intoxicações por fármacos (Pinho et al., 2007; Ware, 2010).

Estudos realizados com grandes felídeos requerem o uso de contenção química devido ao temperamento da espécie em contato com humanos. Os fármacos usados para a contenção desses animais devem agir de forma rápida $\mathrm{e}$ confiável para os manipuladores quando aplicados por via intramuscular, por meio de dardos, e ao mesmo tempo fornecer estabilidade cardiorrespiratória aos animais (Wenger et al., 2010).

Para a contenção química de grandes felinos, fármacos como os alfa-2 agonistas são comumente utilizados, entretanto, quando administrados isoladamente, esses não produzem imobilização confiável em felídeos selvagens por produzirem uma sedação leve que pode levar a um despertar rápido durante a estimulação (Caulkett e Arnemo, 2013). Esta é uma característica que justifica a necessidade de associação de outros fármacos, como a cetamina, aos agonistas $\alpha-2$ adrenérgicos, de modo a produzir inconsciência intensa.

As alterações cardiovasculares esperadas dos agonistas $\alpha-2$ adrenérgicos são bradicardia, bloqueio atrioventricular, redução do débito cardíaco e hipotensão (Golden et al., 1998), enquanto a cetamina provoca efeitos que antagonizam a atividade depressora dos agonistas $\alpha$-2 adrenérgicos, como taquicardia e hipertensão (Lin, 2013). Consequentemente, esses fármacos podem causar alterações eletrocardiográficas relacionadas às modificações que eles causam em todos os sistemas orgânicos.

A associação desses fármacos produz uma sedação de cinco a 20 minutos (Carpenter e Branson, 2013), de modo que procedimentos prolongados em grandes felídeos requerem a manutenção anestésica com anestesia geral inalatória. $\mathrm{O}$ isoflurano e o sevoflurano são os anestésicos de escolha por serem de fácil controle do plano anestésico e por apresentarem recuperação anestésica rápida e baixa taxa de metabolização quando comparados à anestesia intravenosa total (Oliva e Fantoni, 2010; Carpenter e Branson, 2013). Ambos os anestésicos inalatórios são considerados os menos arritmogênicos e não predispõem $\mathrm{o}$ coração à geração de extrassístoles, entretanto esse efeito pode ser exacerbado ao se associarem fármacos agonistas adrenérgicos (Steffey e Mama, 2013).

Considerando a escassez de dados relacionados à realização e interpretação de eletrocardiogramas em grandes felídeos, este trabalho foi escrito com o objetivo de descrever os achados eletrocardiográficos em animais da espécie Puma concolor durante a utilização da cetamina e da detomidina associadas à anestesia geral com isoflurano ou sevoflurano.

\section{MATERIAL E MÉTODOS}

Este estudo foi aprovado pelo Instituto Brasileiro do Meio Ambiente e Recursos Renováveis (IBAMA), sob o protocolo de número-05188761, e pelo Comitê de Ética em Uso de Animais da UFMS, sob o número de registro 628/2014. Foram utilizadas 11 onças-pardas, de idades aproximadas entre quatro e oito anos, e classificadas clinicamente como saudáveis mediante observação da ausência de sinais neurológicos, desidratação, perda de sangue ou diarreia (Gunkel e Lafortune, 2007). As onçaspardas eram mantidas pelo Centro de Reabilitação de Animais Silvestres (CRAS) em Campo Grande, MS, Brasil, e pela fazenda Green Farm $\mathrm{CO}_{2}$ Free, em Itaquiraí, MS, Brasil.

Os felinos foram mantidos em seu cativeiro de origem até o momento dos procedimentos anestésicos e submetidos ao jejum prévio 
alimentar por 12 horas, sem restrição de água. Todos foram manuseados pela mesma equipe, que utilizou os mesmos métodos de manejo e contenção prévia, realizada em caixas de contenção para prevenir a influência de variáveis extras nos resultados.

Devido à natureza selvagem da espécie, as onças foram submetidas à sedação sem avaliação préanestésica. As onças foram alocadas em caixas de contenção mecânica, de modo que permitiu que a contenção química fosse realizada utilizando-se de $0,15 \mathrm{mg} / \mathrm{kg}$ de detomidina $\left(10 \mathrm{mg} / \mathrm{mL}\right.$, Dormium $\mathrm{V}^{\circledR}$, Agener União Saúde Animal, Pouso Alegre, MG, Brasil), associada a $5 \mathrm{mg} / \mathrm{kg}$ de cetamina $\left(100 \mathrm{mg} / \mathrm{mL}\right.$, Vetaset ${ }^{\circledR}$, Fort Dodge Saúde Animal, Campinas/SP, Brasil) por via intramuscular, mediante o uso de dardos remotos (Dist-Inject nylon easy model $5 \mathrm{~mL}$, $11 \mathrm{~mm}$, Switzerland), aplicados por rifle a ar (Syringer projector Dist-Inject model $50 \mathrm{~N}$, Switzerland).

Após 30 minutos, a veia cefálica esquerda foi cateterizada, e a indução anestésica foi realizada com propofol intravenoso na dosagem de $2 \mathrm{mg} / \mathrm{kg}$ $\left(10 \mathrm{mg} / \mathrm{mL}\right.$, Propovan ${ }^{\circledR}$, Cristália, São Paulo, SP, Brasil), de forma a produzir maior relaxamento devido ao seu efeito hipnótico, que facilita a intubação traqueal, realizada com traqueotubo de tamanho 9,5 e os animais foram mantidos anestesiados sob anestesia inalatória a 1 CAM com sevoflurano $\left(\right.$ Sevocris $^{\circledR}$, Cristália, São Paulo/SP, Brasil) ou isoflurano (Isoforine ${ }^{\circledR}$, Cristália, São Paulo/SP, Brasil), utilizando-se oxigênio $100 \%$. O grupo anestesiado com sevoflurano (GSEVO) incluiu seis onças, e o grupo do isoflurano (GISO) foi composto por cinco animais, alocado aleatoriamente. Para registro eletrocardiográfico, os animais foram mantidos em decúbito lateral direito (Larsson et al., 2008) e os eletrodos do ECG foram posicionados em contato com a pele em pontos específicos: membros torácicos direito e esquerdo próximo ao olecrano; e nos membros pélvicos direito e esquerdo acima do ligamento patelar. Os contatos dos eletrodos foram umedecidos com álcool.

Decorridos 10 minutos da indução anestésica, iniciaram-se os registros a uma sensitividade em posição $\mathrm{N}=1$ e velocidade de $50 \mathrm{~mm} / \mathrm{s}$ em eletrocardiografia computadorizada $\left(E C G-P^{\circledR}\right.$ Tecnologia Eletrônica Brasileira - TEB). Os registros eletrocardiográficos foram realizados nas derivações bipolares DI, DII, DIII e nas derivações calculadas unipolares aVR, aVL e aVF. Avaliaram-se a frequência e o ritmo cardíacos, eixo médio elétrico e a amplitude e duração das ondas P, QRS e T; e dos intervalos PR, ST e QT. As análises dos registros eletrocardiográficos foram realizadas por 60 minutos, enquanto os animais estavam no primeiro plano do III estágio anestésico (Massone, 1999).

\section{RESULTADOS}

Os traçados eletrocardiográficos das onças-pardas anestesiadas com sevoflurano e isoflurano estão representados nas Fig. 1 e 2, respectivamente. Os parâmetros eletrocardiográficos individuais estão descritos nas Tab. 1 e 2. As frequências cardíacas dos animais variaram entre 78 e $116 \mathrm{bpm}$ no GSEVO e entre 80 e $113 \mathrm{bpm}$ no GISO, no qual se obteve uma média de $95 \pm 13$.

O ritmo cardíaco dos animais foi considerado sinusal pela presença de onda $\mathrm{P}$ na maioria dos traçados, com exceção de três animais. A onça 2 do GSEVO manteve ritmo sinoventricular irregular durante todo o período avaliado e apresentou complexo QRS largo e bizarro durante os primeiros 15 minutos da monitoração (Fig. 1, onça 2A), seguidos da redução da duração do complexo QRS, que deixou de ter aparência bizarra. A onça 6 do GSEVO apresentou ritmo sinusal (Fig. 1, onça 6A) passando para ritmo sinoventricular com parada atrial por ausência de onda P (Fig. 1, onça 6B). Outro distúrbio de ritmo avaliado ocorreu na onça 2 do GISO (Fig. 2), que apresentou bloqueio de ramo direito do feixe de His.

O animal 1 do GSEVO apresentou um episódio isolado de extrassístole ventricular durante a monitoração. Já nas onças 1, 3, 4 e 5 do GSEVO foi detectado bloqueio atrioventricular de primeiro grau (BAV $1^{\circ}$ grau). A onça 3 do GSEVO apresentou o complexo QRS com amplitude diminuída e onda $\mathrm{S}$ profunda, enquanto a onça 4 do GISO apresentou um episódio de complexo atrial prematuro.

Os traçados das onças 5 e 6 do GSEVO e das onças 3 e 5 do GISO apresentaram-se com a onda $\mathrm{T}$ com amplitude aumentada e em tenda. 


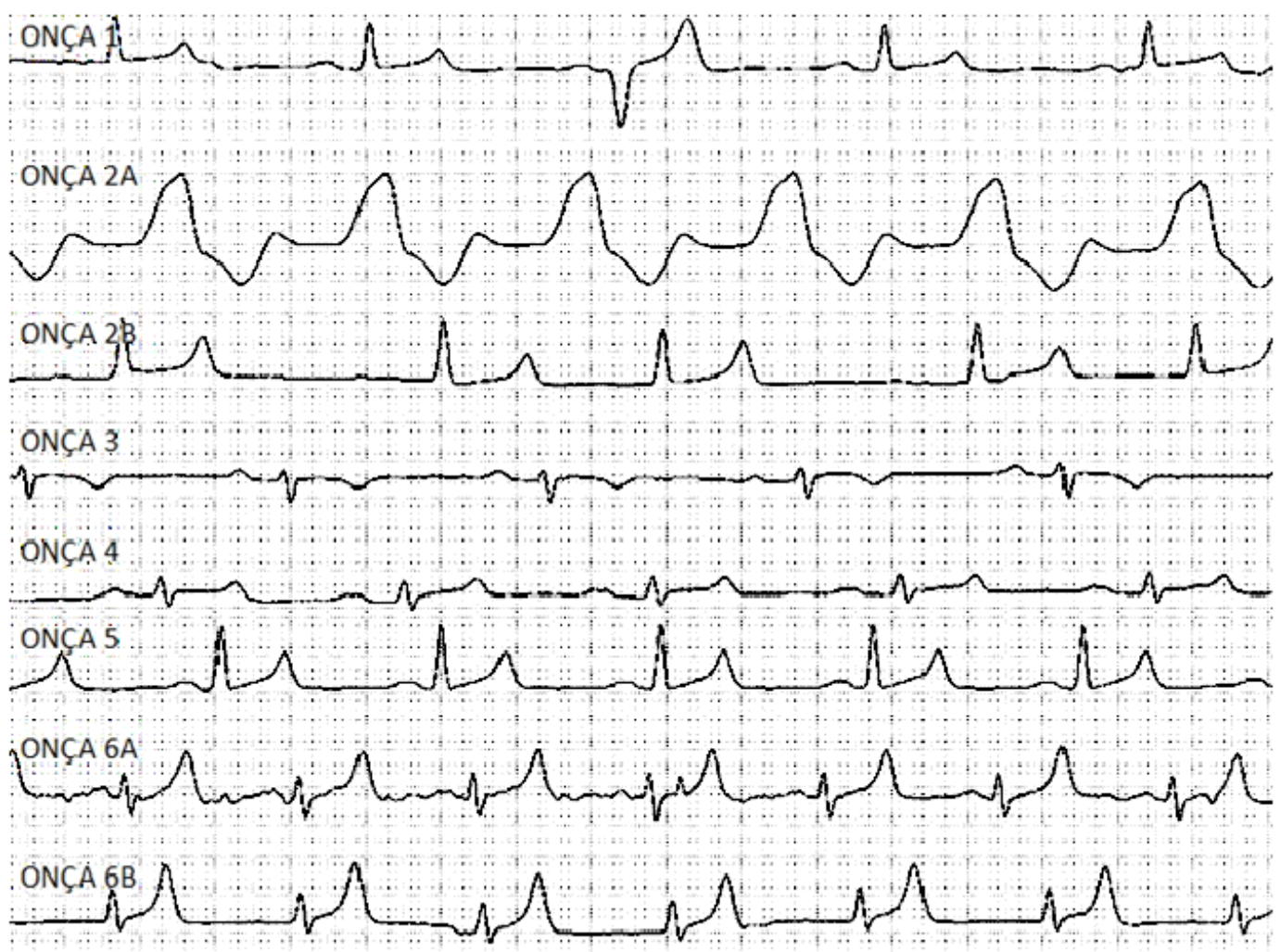

Figura 1. Traçados eletrocardiográficos das onças-pardas anestesiadas com sevoflurano (GSEVO).

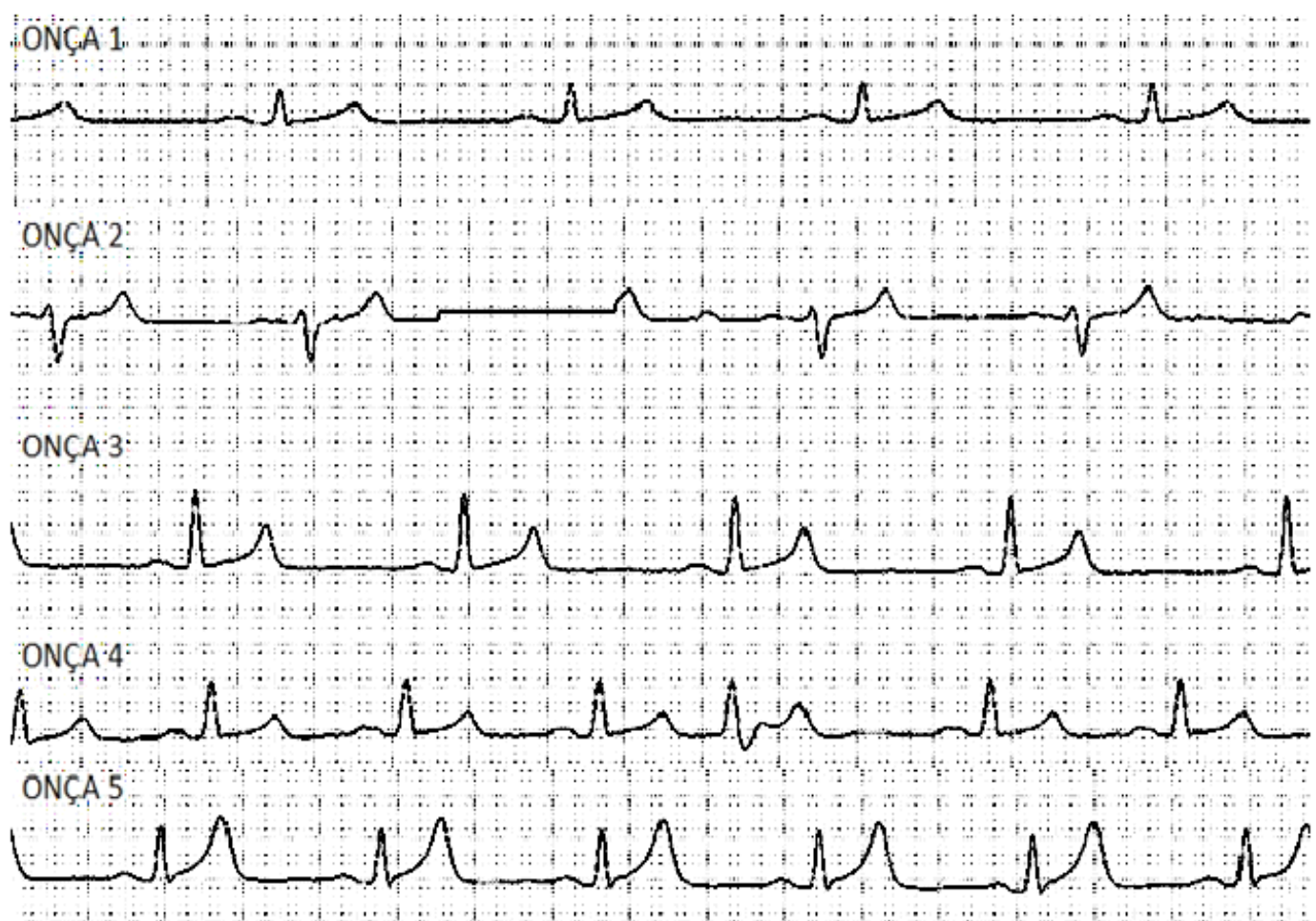

Figura 2. Traçado eletrocardiográfico das onças-pardas anestesiadas com isoflurano (GISO). 
O eixo elétrico cardíaco apresentou-se alterado com desvio à esquerda para a onça 4 e com desvio à direita para a onça 5 , ambas do GSEVO (Tab. 1). O eixo elétrico da onça 5 do GISO não pôde ser mensurado devido a interferências elétricas em algumas derivações. O eixo cardíaco dos demais animais de ambos os grupos foi de $0^{\circ}$ a $160^{\circ}$ (Tab. 1 e 2 ).

Tabela 1. Descrição dos parâmetros eletrocardiográficos individuais dos animais anestesiados com sevoflurano (GSEVO)

\begin{tabular}{ccccccc}
\hline Animal & 1 & 2 & 3 & 4 & 5 & 6 \\
\hline FC (bpm) & 87 & 90 & 78 & 94 & 106 & 116 \\
Eixo $\left(^{\circ}\right)$ & $60-90+$ & $60-90+$ & $30-60+$ & $60-90-$ & $90-120-$ & $60-90+$ \\
Amplitude da P (mV) & 0,1 & - & 0,09 & 0,08 & 0,1 & 0,1 \\
Duração da P (ms) & 77 & - & 80 & 83 & 83 & 60 \\
Intervalo PR (ms) & 140 & - & 160 & 153 & 113 & 90 \\
Amplitude QRS (mV) & 0,72 & 0,78 & 0,18 & 0,24 & 0,84 & 0,3 \\
Duração QRS (ms) & 57 & 63 & 60 & 67 & 57 & 50 \\
Intervalo QT (ms) & 260 & 293 & 270 & 257 & 240 & 233 \\
Onda T (mV) & $0,27+$ & $0,47+$ & $0,26-$ & $0,24+$ & $0,5+$ & $0,76+$ \\
\hline
\end{tabular}

Tabela 2. Descrição dos parâmetros eletrocardiográficos individuais dos animais anestesiados com isoflurano (GISO)

\begin{tabular}{cccccc}
\hline Animal & 1 & 2 & 3 & 4 & 5 \\
\hline FC (bpm) $_{\text {Eixo }\left({ }^{\circ}\right)}$ & 80 & 87 & 86 & 113 & 106 \\
Amplitude da P (mV) & $60-90+$ & $60-90+$ & $30-60+$ & $60-90+$ & - \\
Duração da P (ms) & 0,08 & 0,08 & 0,1 & 0,09 & 0,09 \\
Intervalo PR (ms) & 73 & 80 & 80 & 70 & 73 \\
Amplitude QRS (mV) & 137 & 100 & 113 & 103 & 107 \\
Duração QRS (ms) & 0,64 & 0,14 & 0,94 & 0,65 & 0,67 \\
Intervalo QT (ms) & 47 & 70 & 50 & 63 & 60 \\
Onda T (mV) & 280 & 257 & 243 & 237 & 240 \\
& $0,14+$ & $0,3+$ & $0,51+$ & $0,27+$ & $0,78+$ \\
\hline
\end{tabular}

\section{DISCUSSÃO}

As frequências cardíacas das onças-pardas anestesiadas neste estudo mantiveram-se entre 78 e 116 batimentos por minuto (bpm), valores considerados normais para grandes felídeos, que devem variar entre 70 e $140 \mathrm{bpm}$ (Deem e Karesh, 2001). Alguns autores estimam a frequência cardíaca para grandes felinos por meio da escala alométrica, na qual mamíferos que pesam de 100 a $250 \mathrm{~kg}$ devem possuir a frequência cardíaca entre 60 e $80 \mathrm{bpm}$ (Sedgwick, 1991).

A maioria dos felídeos anestesiados neste estudo apresentou ritmo cardíaco sinusal, considerado normal para todos os mamíferos. Entretanto, as onças 2 e 6 do grupo GSEVO apresentaram uma parada atrial (atrial standstill) com condução sinoventricular. $\mathrm{O}$ atrial standstill ocorre pelo bloqueio da despolarização atrial, causada principalmente por hipercalemia no paciente ${ }^{8}$.
Representado pela ausência de onda $\mathrm{P}$ no eletrocardiograma, o atrial standstill pode estar acompanhado do aumento progressivo da duração do complexo QRS e do aumento da amplitude da onda $\mathrm{T}$, sendo este um dado encontrado na onça 6. Além da hipercalemia, outras condições podem fazer com que esse traçado seja observado, entre elas insuficiência renal, cetoacidose diabética, acidose metabólica, hipoadrenocorticismo e até mesmo as próprias cardiomiopatias em gatos (Fillipi, 2011). Não há evidências de que esses distúrbios de ritmo sejam causados pela utilização de anestésicos.

Alteração eletrocardiográfica, como extrassístole ventricular, encontrada em um animal do GSEVO, é considerada a arritmia mais comum relatada em cães e gatos. Ela ocorre quando o impulso gerado caminha pelas fibras de Purkinje da musculatura ventricular em vez de seguir pelo sistema especializado de condução, o que leva à incapacidade da onda de ativação de penetrar no 
nó atrioventricular e despolarizar o nó sinusal (Fillipi, 2011). As causas mais comuns descritas em felinos são as doenças primárias do miocárdio e a reação do miocárdio a diferentes drogas, como as anestésicas (Goodwin, 2002).

Apesar de não aumentar a sensibilidade do miocárdio às catecolaminas, a cetamina é um anestésico que causa estimulação indireta do sistema cardiovascular pela inibição da recaptação de catecolaminas pelos terminais nervosos, causando um efeito inotrópico sobre o miocárdio (Lin, 2013). Devido à sua atividade arritmogênica comprovada (Hikasa et al., 1996) e considerando-se o seu período de ação de aproximadamente 30 a 40 minutos (Stewart, 1999), podem-se associar as extrassístoles ventriculares encontradas neste estudo ao uso deste fármaco.

A sensibilidade do miocárdio às catecolaminas, ao se utilizarem anestésicos inalatórios como o halotano, era uma preocupação constante devido à sua capacidade arritmogênica, na qual extrassístoles ventriculares são induzidas pela depressão do nó sinusal e pela desaceleração nodal atrioventricular (Hikasa et al., 1996; Oliva e Fantoni, 2010). Entretanto, o isoflurano é um anestésico que não sensibiliza o miocárdio à ação da adrenalina, exceto em casos em que a concentração do anestésico é elevada rapidamente (Oliva e Fantoni, 2010), fato este que não pode ser associado ao estudo, devido à utilização de 1CAM tanto para o isoflurano quanto para o sevoflurano, concentração esta considerada superficial para anestesia inalatória. $\mathrm{O}$ isoflurano e o sevoflurano não apresentam diferenças significativas quanto à capacidade de sensibilizar o miocárdio de felinos domésticos às catecolaminas (Hikasa et al., 1996), podendo-se presumir que, no presente estudo, os transtornos arritmogênicos encontrados nas onças anestesiadas não estão associados ao uso dos anestésicos inalatórios.

Apesar do bom estado de sedação dos felinos após o uso dos anestésicos intramusculares, o propofol foi utilizado em dose mínima recomendada, conforme Zoran (1993), para indução anestésica, de modo a facilitar a intubação orotraqueal por produzir grande relaxamento laríngeo (Cleale et al., 2009). A dose mínima recomendada de $2 \mathrm{mg} / \mathrm{kg}$ foi suficiente para intubação traqueal quando comparada à dose de $7 \mathrm{mg} / \mathrm{kg}$ utilizada por Cleale et al. (2009) para indução e intubação orotraqueal em felinos domésticos sem a utilização de pré-anestésicos. Pode-se, desse modo, atribuir que, devido à reduzida dose de propofol utilizada, seus efeitos adversos causados de forma dose-dependente, como aumento do intervalo PR, hipotensão e redução da frequência cardíaca (Pereira et al., 2004), não puderam ser correlacionados aos achados eletrocardiográficos neste estudo.

O intervalo PR em felinos domésticos deve estar aumentado para que haja o BAV de primeiro grau (Fillipi, 2011), corroborando os achados eletrocardiográficos dos animais 1, 2, 3, 4 e 5 do GSEVO e do GISO. Sua ocorrência isoladamente não tem significado clínico, servindo apenas de um indicador precoce de disfunção no nodo atrioventricular (Goodwin, 2002). Essas alterações podem ser associadas a distúrbios eletrolíticos, como hipo e hipercalemia (Fillipi, 2011), e também ao uso de agonistas alfa-2 adrenérgicos que inibem o tônus simpático devido à redução da liberação pré-sináptica de noradrenalina, levando a alterações como bradicardia, redução de débito cardíaco e bloqueios atrioventriculares de primeiro e segundo graus (Murrel e Hellebrekers, 2005). Apesar dos efeitos depressores dos alfa-2 adrenérgicos, a frequência cardíaca manteve-se normal para a espécie, fator este que pode estar relacionado aos efeitos estimulantes da cetamina. Dessa forma, os BAVs não podem ser diretamente associados ao uso da detomidina. $\mathrm{O}$ eletrocardiograma normal dos grandes felídeos é afetado pelo uso dessas drogas, entretanto não é possível obter os traçados eletrocardiográficos sem que esses animais sejam anestesiados (Lloyd, 2009). Assim, tanto os efeitos sedativos quanto os efeitos adversos da detomidina ainda estavam presentes durante a avaliação eletrocardiográfica dos animais em questão, considerando-se que o período hábil desse fármaco, quando relatado em outras espécies, pode chegar a 60 minutos (Cortopassi e Fantoni, 2010).

O bloqueio do ramo direito do feixe de His é uma condição que, em felinos domésticos, está associada à cardiomiopatias, hipercalcemia e neoplasias cardíacas. Nesse caso, achados eletrocardiográficos são representados por um complexo QRS maior do que 60ms (Fillipi, 
2011), corroborando o resultado encontrado na onça 2 do GISO. Essa condição está associada ao desvio de eixo médio para a direita.

Segundo Goodwin (2002), o eixo cardíaco esperado de gatos domésticos saudáveis encontra-se entre $0^{\circ}$ e $160^{\circ}$. O eixo descrito para outras espécies de felinos de grande porte - leão e tigre - varia de $+60^{\circ}$ a $+90^{\circ}$ (Larsson et al., 2008). Dados na literatura indicam que, na onçaparda, o eixo varia entre +36 e $41^{\circ}$, porém esse valor foi encontrado em apenas um exemplar (Adania et al., 2007). Entretanto, pode-se afirmar que apenas o animal 5 do GSEVO apresentou desvio de eixo à direita, com os valores variando entre $-90^{\circ}$ e $-120^{\circ}$, o que pode ser um indicativo de hipertrofia de ventrículo direito, enquanto o animal 4 do GSEVO, que apresentou desvio de eixo à esquerda, com valores variando entre $-90^{\circ}$ e $-60^{\circ}$, pode ter uma hipertrofia de ventrículo esquerdo (Fillipi, 2011).

O desequilíbrio de eletrólitos acarreta efeitos cardiotóxicos graves e algumas vezes letais. Ocorre a diminuição da excitabilidade e o aumento do período refratário do músculo, além da diminuição da velocidade de condução (Fillipi, 2011). Os animais 5 e 6 do GSEVO e 3 e 5 do GISO apresentaram alterações em amplitude e tempo da onda $T$. Isso pode ser explicado por alterações eletrolíticas, principalmente pela hipercalemia. As principais alterações decorrentes da hipercalemia são o desaparecimento da onda P (átrio silencioso) e o aumento da onda $\mathrm{T}$, muitas vezes em tenda (Pinho et al., 2007). Entretanto, já foi relatado o aumento da onda $\mathrm{T}$ sem que houvesse alterações bioquímicas do potássio sérico em grandes felídeos selvagens (Reilly et al., 2014).

A análise eletrocardiográfica de grandes felídeos é possível desde que os animais sejam mantidos anestesiados. Entretanto, o uso de diferentes tipos de anestesia pode ocasionar alterações no ECG difíceis de serem correlacionadas à normalidade nessas espécies, apesar de esse estudo mostrar os achados esperados para os protocolos usados. Portanto, mais estudos eletrocardiográficos são necessários, em uma tentativa de correlacionar os atuais achados com parâmetros normais ou alterados pelo uso de sedativos e anestesia geral.

\section{AGRADECIMENTOS}

Os autores agradecem a todos os funcionários do Centro de Reabilitação de Animais Silvestres de Campo Grande e à Green Farm CO2 Free, de Itaquiraí, que recebeu os investigadores e ofereceu sua atenção irrestrita. Os agradecimentos se estendem também ao ICMbio, por sua assistência e físcalização dos procedimentos neste projeto.

\section{REFERÊNCIAS}

ADANIA, C.H.; SILVA, J.C.R.; FELIPPE, P.A.N. Carnivora: felidae (onça suçuarana, jaguatirica e gato-do-mato) In: CUBAS, Z.S.; RAMOS, J.; CANTÃO-DIAS, J.L.C. (Eds.). Tratado de animais selvagens medicina veterinária. São Paulo: Roca, 2014. p.779-818.

CARPENTER, R.E.; BRANSON, D.B. Animais de Zoológico e Exóticos. In: TRANQUILLI, W.J.; THURMON, J.C.; GRIMM, K.A. (Eds.). Anestesiologia e analgesia veterinária. São Paulo: Roca, 2013. p.860-883.

CAULKETT, N.A.; ARNEMO, J.M. Imobilização química de mamíferos terrestres de vida livre. In: TRANQUILLI, W.J.; THURMON, J.C.; GRIMM, K.A. (Eds.). Anestesiologia e analgesia veterinária. São Paulo: Roca, 2013. p.884-913.

CLEALE, R.M.; MUIR, W.W.; WASELAU, A.C. et al. Pharmacokinetic and phamacodynamic evaluation of propofol administered to cats in a novel aqueour, nanodroplet formulation or as na oil-in-water macroemulsion. Vet. Pharmacol. Ther., v.32, p.436-445, 2009.

CORTOPASSI, S.R.G.; FANTONI, D.T. Medicação pré-anestésica. In: __ (Eds.). Anestesia em cães e gatos. São Paulo: Roca, 2010. p.217-227.

DEEM, S.L.; KARESH, W. Manual del programa de salud del jaguar. [Bern]: Wildlife Conservation Society, 2001. 56p.

FILLIPI, L.H. (Ed.). O eletrocardiograma na medicina veterinária. São Paulo: Roca, 2011. $242 p$. 
GOLDEN, A.L.; BRIGHT, M.; DANIEL, B. et al. Cardiovascular effects of the a2-adrenergic receptor agonist medetomidine in clinically normal cats anesthetized with isoflurane. Am. J. Vet. Res., v.59, p.509-513, 1998.

GOODWIN, J.K. Eletrocardiografia. In: TILLEY, L.P.; GOODWIN, J.K. (Eds.). Manual de cardiologia para cães e gatos. São Paulo: Roca, 2002. p.39-65.

GUNKEL, C.; LAFORTUNE, M. Felids. In: WEST, G.; HEARD, D.; CAULKETT, N. (Eds.). Zoo animal \& wildlife immobilization and anesthesia. Oxford: Blackwell Publishing, 2007. p.443-458.

HIKASA, Y.; OKABE, K.; TAKASE, S.; OGASAWARA, S. Ventricular arrhytmogenic dose of adrenaline during sevoflurane, isoflurane, and halothane anaesthesia either with or without ketamine or thiopentone in cats. Res. Vet. Sci., v.60, p.134-137, 1996.

LARSSON, M.H.M.A.; COELHO, F.M.; OLIVEIRA, V.M.C. et al. Electrocardiographic parameters of capitive lions (Panthera leon) and tigers (Panthera tigris) immobilized with ketamine plus xylazine. J. Zoo Wildl. Med., v.39, p.314-319, 2008.

LIN, H.C. Anestésicos dissociativos. In: TRANQUILLI, W.J.; THURMON, J.C.; GRIMM, K.A. (Eds.). Anestesiologia e analgesia veterinária. São Paulo: Roca, 2013. p.335-384.

LLOYD, C. Eletrocardiographic parameters of captive Arabian leopards (Panthera pardusnimr) immobilized with medetomidine and ketamine. Wildl. Life Middle East, v.3, [p.7], 2009.

MASSONE, F. (Ed.). Anestesiologia veterinária: farmacologia e técnicas. Rio de Janeiro: Guanabara, 1999. 225p.

MURRELL, J.C.; HELLBREKERS, L.J. Medetomidine and dexmedetomidine: a review of cardiovascular effects and antinociceptive properties in the dog. Vet. Anaesth. Analg., v.32, p.117-127, 2005.

ODA, S.G.S.; LEOMIL NETO, M.; FEDULLO, J.D.L. et al. Padronização de alguns parâmetros eletrocardiográficos de animais da espécie Herpailurus yagouaroundi, mantidos em cativeiro. Rev. Bras. Ciênc. Vet., v.14, p.47-50, 2007.
OLIVA, V.N.L.S.; FANTONI, D.T. Anestesia inalatória. In: FANTONI, D.T.; CORTIPASSI, S.R.G. (Eds.). Anestesia em cães e gatos. São Paulo: Roca, 2010. p.246-258.

PEREIRA, G.G.; LARSSON, M.A.; YAMAKI, F.L. et al. Effects of propofol on electrocardiogram and systolic blood pressure od healthy cats pré-medicated with acepromazine. Vet. Anaesth. Analg., v.31, p.235-238, 2004.

PINHO, J.A.; BACELLAR, M.S.C.; RIBEIRO, D.G.L.; DARWICH, R.N. et al. Diretrizes de interpretação de eletrocardiograma de repouso. Arq. Bras. Cardiol., v.80, p.4-18, 2007.

REILLY, S.; SEDDIGHI, M.R.; STEEL, J.C. et al. Selected clinical, biochemical, and electrolyte alteration in anesthetizes captive tigers (Panthera tigris) and lions (Panthera leo). J. Zoo Wildl. Med., v.45, p.328-334, 2014.

SEDGWICK, C.J. Allometrically scaling the database for vital sign assessment used in general anesthesia of zoological species. Proc. Am. Assoc. Zoo Vet., 1991. p.360-369.

STEFFEY, E.P.; MAMA, K.R. Anestésicos inalatórios. In: TRANQUILLI, W.J.; THURMON, J.C.; GRIMM, K.A. (Eds.). Anestesiologia e analgesia veterinária. São Paulo: Roca, 2013. p.335-384.

STEWARD, A.B. Ketamine: review of its pharmacology and use in pediatric anesthesia. Anesth. Prog., v.46, p.10-20, 1999.

WARE, W.A. Testes diagnósticos para o sistema cardiovascular. In: NELSON, R.W.; COUTO, C.G. (Eds.). Medicina interna de pequenos animais. São Paulo: Elsevier, 2010. p.16-32.

WENGER, S.; BUSS, P.; JOUBERT, J. et al. Evaluation of butorphanol, medetomidine and midazolam as a reversible narcotic combination in free-ranging African lions (Panthera leo). Vet. Anaesth. Analg., v.37, p.491-500, 2010.

ZORAN, D.L.; RIEDESEL, D.H.; DYER, D.C. Pharmacokinetics of propofol in mixed breed dogs and greyhounds. Am. J. Vet. Res., v.54, p.755, 1993. 